\title{
Numerical Analysis of Surface Force of Diamond-Like Carbon Surface Coated with Monolayer Lubricant Film
}

\author{
Kyosuke Ono* \\ Tokyo Institute of Technology, Ookayama, Meguro-ku, Tokyo, Japan \\ *Corresponding author: Kyosuke Ono (Ono_kyosuke@nifty.com)
}

Manuscript received 11 October 2017; accepted 21 July 2018; published 31 December 2018

Presented at the 6th World Tribology Congress 2017 Beijing, September 2017

\begin{abstract}
Diamond-like carbon (DLC) is widely used as a hard, protective layer with a relatively low surface energy. In the head-disk interface in magnetic disk drives, however, the DLC layer is coated with a monolayer perfluoropolyether lubricant with a high bond ratio to avoid DLC-DLC contact and to secure head/disk wear reliability. In this study, we theoretically analyzed the effect of lubricant thickness and bond ratio on the adhesion force between the head-disk interface (HDI) in a mono/submono-layer thickness regime. It was found that the adhesion force had the lowest sensitivity to lubricant thickness variations at a 0.85 bond ratio. In addition, the maximum adhesion force was minimized when the lubricant thickness was $\sim 0.6 \mathrm{~nm}$ for the measured parameter values of the HDI. This suggests that the current lubricant thickness of 1.0-1.2 $\mathrm{nm}$ can be reduced to $0.6 \mathrm{~nm}$, accompanied by a slight decrease in the adhesion force and a slight increase in the resistance against any variation in its thickness. This tribo-surface-modification concept can be applied to surfacemodification coatings in other fields such as micro/nano-electromechanical systems. The compatibility of the theoretical surface energy function with experimental data indicates the validity and consistency of this theory.
\end{abstract}

\section{Keywords}

surface force analysis, rough surface adhesive contact, asperity meniscus pressure, van der Waals pressure, head-disk interface, diamond-like carbon, molecularly thin lubricant film, bond ratio, surface-modification coating

\section{Introduction}

The mechanical reliability of head slider flying on a moving disk surface with only $0.7 \mathrm{~nm}$ clearance is secured by a molecularly thin perfluoropolyether (PFPE) lubricant film coated on a few-nanometers-thick diamond-like carbon (DLC) layer on the disk. The head surface is also coated with a DLC layer a few nanometers thick. By means of the thermal flying height control (TFC) technique, a small head area on the trailing air-bearing pad protrudes to the high-speed disk surface at a subnanometer clearance only during the read/write operation [1]. Clearance is practically defined as the back-off distance of the head apex from the position of contact detection with the lubricant. Although the contact position is not clear in terms of separation from the mean asperity height of a mobile or bonded lubricant surface, we consider here that clearance is the distance between the head apex and the surface height of $3 \sigma_{a}$ from the mean asperity height of the lubricant surface. To increase the recording density, great efforts to further reduce the clearance down to $\sim 0.5 \mathrm{~nm}$ are being made [2]. At such small head flying heights (FH:FH is defined as the distance between the mean height of the lubricant surface and the head apex), because the mobile and bonded lubricant on high asperities are intermittently in contact with the head surface, the increment of attractive force can overcome that of the air-bearing force, resulting in an unstable FH region of the resultant force. For this reason, peculiar phenomena such as jumps between the asperity contact state and the flying state occur as the head surface protrudes to the disk [3]. Moreover, in a slight lubricant contact region, drastically large spacing variations having lower-frequency components of less than $100 \mathrm{kHz}$ can appear when the microwaviness of the disk surface is increased to some extent [2].

To elucidate these peculiar phenomena, it is essential to evaluate the rigorous surface attractive pressure between the head and disk surfaces considering the real surface texture parameters of the disk and head. For this purpose, we previously characterized the texture parameters of commercially used perpendicular disks and recording heads [4]. Then, we formulated the surface adhesive pressure generated from high-density small asperities covered with a DLC layer and a lubricant film whose main fraction was bonded to the DLC surface and had a thin mobile layer. By applying a theoretically derived surface force to a spherically protruded head surface 
and a simple slider model of a single degree of freedom, we theoretically revealed the mechanism of the abovementioned phenomena [5]. Moreover, we demonstrated that this theory can explain the difference of touchdown behaviors of commercially available head sliders at inner, middle, and outer radius positions of the disk [6,7]. In these studies, we calculated the surface force only for the specific lubricant thickness and bond ratio of commercially available disk surfaces.

To reduce the spacing between the recording head and the magnetic medium, it is important to reduce the lubricant thickness, as well as the FH and DLC layer thicknesses. In near-contact and asperity contact regimes, it is important to reduce the surface adhesive force because normal and shear contact stresses are mainly influenced by the surface adhesive force. Therefore, it will be valuable to investigate the general characteristics of the attractive pressure of a disk surface with a molecularly thin lubricant with different bond ratios coated onto the DLC underlayer and to clarify how the lubricant thickness can be further reduced without increasing the attractive pressure. The mathematical formulae derived in this paper will provide a powerful tool to evaluate and design a smooth and molecularly thin lubricated surface with reduced adhesive force for use in other engineering fields such as micro/ nano-electromechanical systems.

The surface energy function with respect to lubricant thickness and bond ratio is next derived from the surface attractive pressure equations. Good compatibility of this surface energy function with experimental data would suggest the validity and consistency of this surface force theory.

2 Theory of adhesion pressure and surface force between a spherical head and a rough disk surface coated with DLC and bonded and mobile lubricant layers

2.1 Rough surface contact model and spherical head-disk contact model

Figure 1(a) shows an analytical model of the asperity contact between the thermally protruded head and the magnetic disk. It is well known that contact between two rough surfaces can be modeled as contact between a smooth surface and a rough surface with a composite roughness. According to our measurements, however, the mean asperity height of the head surface was approximately half that of the perpendicular recording disk, and the mean asperity radius was ten times larger than that of the disk. Therefore, in the present headdisk interface, the head surface can be considered to be almost smooth compared to the disk surface. A molecularly thin lubricant film is coated over the DLC layer on the perpendicular magnetic medium. The head-disk spacing (HDS) between the head apex and the mean surface height of the bonded lubricant surface (BLS) is denoted by $h_{b l}$, and the distance between the head apex and the mean asperity height of the BLS is termed the head separation, denoted by $d_{b l}$. The nondimensional separation $d_{b l} / \sigma_{a}$ [where $\sigma_{a}$ is the root-mean-square (rms) value of the asperity height. See Fig. 1(b)] is often used for illustration using the same terminology as separation for simplicity. The separation of head apex $d_{m l}$ from the mean asperity height of the mobile lubricant surface (MLS) and $d_{m l} / \sigma_{a}$ are also used in this paper. The distance of the mean asperity height from the mean surface height of the BLS is denoted by $z_{m}$.

Figure 1(b) shows the local asperity contact model for the head and disk surfaces. The local HDS between the local head and the mean height of the BLS is denoted by $h_{a}$, whereas the local separation from the mean asperity height of the BLS is denoted by $d_{a}\left(d_{a}=h_{a}-z_{m}\right)$. The height of the asperity peak above the mean asperity height is denoted by $z_{a}$. The total lubricant thickness, bonded lubricant thickness, and average mobile lubricant thickness are denoted by $T_{L}, T_{b l}$, and $T_{m l}$, respectively. The bond ratio, $B R$, is defined as $B R=T_{b l} / T_{L}$. When the head contacts the disk lightly, upon increasing the TFC power, the smooth head surface contacts the MLS on the asperity summit first, forming an asperity meniscus. Then, it elastically contacts the BLS rather than the DLC surface (DLCS) except for a special case of $T_{b l}=0$.

2.2 Surface force models and assumptions for numerical analysis

The adhesion of a rough surface with a thin lubricant film was first analyzed by Stanley et al. [8] based on the sub-boundary lubrication (SBL) model and the DerjaguinMuller-Toporov [9] adhesion theory using the intermolecular pressure derived from the Lennard-Jones potential (LJP). The SBL model assumes that the thin lubricant layer remains a uniform film of quasi-solid nature with the surface energy of the bulk lubricant that does not form a meniscus bridge at asperity contact. Therefore, it is assumed that, in addition to the lubricant adhesion force at asperity contact, an additional surface force is generated outside the asperity contact area following the method given by Muller et al. [10]. This rough surface adhesion model and analytical method were extensively used for the surface force of the head-disk interface to clarify the static instability of head sliders encountered in the process of reducing the flying height to less than $10 \mathrm{~nm}$ [11-13]. After a TFC head slider was introduced, Vakis et al. [14] and Zheng and Bogy $[15,16]$ evaluated the surface force based on the Stanley-

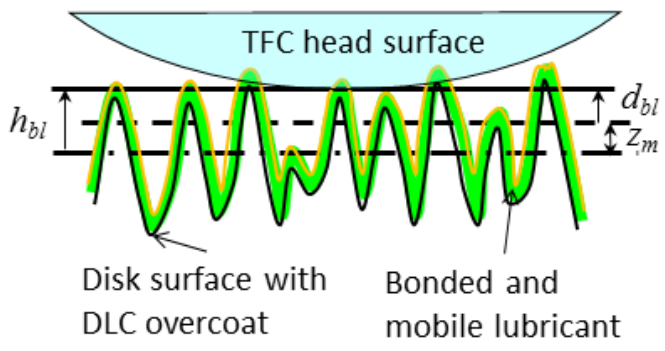

(a) TFC head/disk contact model

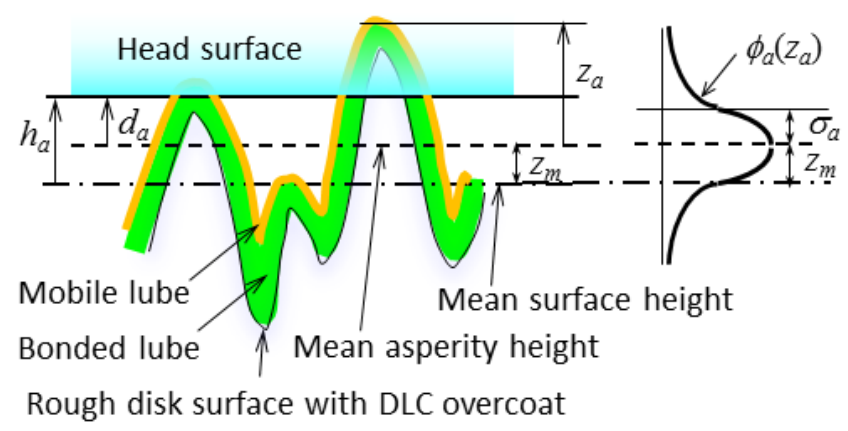

(b) Rough surface contact model

Fig. 1 Rough surface adhesion contact models: (a) TFC head/disk contact model, (b) Rough surface contact model 
Etsion-Bogy (SEB) theory [8] and analyzed the dynamics of the TFC head slider at touchdown. However, they could not elucidate the peculiar phenomena stated above.

In the present rough surface adhesion pressure theory, we employ the surface force theory at noncontacting and contacting asperities derived from the LJP and the GreenwoodWilliamson (GW) asperity contact model [17], similar to the SEB theory. However, we assume dynamic asperity meniscus bridge formation from our experimental evidence described below and ignore the surface force from outside the asperity meniscus contact area. Moreover, we consider the surface force from the DLC underlying the molecularly thin lubricant film, in addition to the surface force from the lubricant film.

The assumption of toe-dipping meniscus formation around small contacting asperities, particularly between a head surface and a moving disk surface, is not easily accepted. However, we experimentally observed the bulk meniscus formation between a bouncing glass sphere with a radius of $1 \mathrm{~mm}$ on a stationary disk surface coated with a 0.69 -nm-thick mobile lubricant film during only a 14- $\mu$ s collision time [18]. From the diffusion equation of a thin liquid film governed by the disjoining pressure, the diffusion speed between a certain distance was found to be proportional to the square of the distance $[19,20]$. Because the square of the associated meniscus radius on an asperity is proportional to the asperity radius and lubricant thickness, it is inferred that a meniscus around an asperity with a radius of $15 \mathrm{~nm}$ can form during only $0.21 \mathrm{~ns}$ at most. During this period, the disk surface at a speed of $20 \mathrm{~m} / \mathrm{s}$ moves only 4 $\mathrm{nm}$, which is less than the asperity meniscus diameter. If we further consider a lowered meniscus height at a 15-nm-radius asperity compared with that at a $1-\mathrm{mm}$-radius sphere, the asperity meniscus can form within only $20 \mathrm{ps}$, during which the disk surface movement is less than $1 \mathrm{~nm}$, even at a $40-\mathrm{m} / \mathrm{s}$ disk speed.

Experimental evidence of the formation of a macroscopic lubricant bridge between the head surface and the spinning disk was also reported in some papers [21, 22]. The lubricant pickup and transfer to the head surface caused by van der Waals forces have been big obstacles to achieving a smaller flying height [23, 24]. Saeki et al. [25] theoretically revealed the tendency of the speed of lubricant bridge formation at an asperity to increase as the asperity radius decreases. This combined evidence suggests the possibility of meniscus formation around asperities at the instant of contact. The assumption of the rapid formation of an asperity meniscus between the head surface and the moving disk surface is also considered rational from the fact that the present theoretical surface force could evaluate the experimental slider dynamics at touchdown $[6,7]$.

The gap between the head surface and the contacting asperity at the periphery of the meniscus is more than $2 T_{m l}$ at the instant of meniscus formation, and the gap will become larger than $1 \mathrm{~nm}$ because of the additional effect of lubricant inflow generated from the difference of the Laplace pressure and disjoining pressure [26]. This suggests that the surface force due to the surface energy from the area outside the meniscus can be negligibly small. Moreover, according to the author's numerical analysis of the surface force at the elastic contact between a $1-\mu \mathrm{m}$-radius sphere and a flat surface without lubricant [27], it was found that the surface force outside the contact area was always approximated by $4 \pi R_{a} \gamma_{s}$, in contrast to the SEB theory. This is because the gap between the asperity and the flat surface outside the adhesive contact area was significantly greater than that in Hertzian contact. Therefore, the adhesion force between the head surface and the contacting asperity can be always expressed as $4 \pi R_{a} \gamma_{l}$ in both cases with and without a mobile lubricant film.

Attractive pressures due to van der Waals (vdW) forces acting on a flying head slider have also been discussed as a cause of the static instability of pico-sliders in the sub-5-nm flying height regime [28-31]. In these theories, the vdW pressure is derived from only the attractive component in the LJP law. The sum of the vdW pressures between multilayers in the HDI by using Hamaker constants can be calculated from Lifshitz theory [32]. However, Lifshitz theory assumes a continuous medium model with multilayer molecules and cannot treat polar surface energy [33]. Therefore, it is not appropriate to use Hamaker constants in this analysis. Thus, we use the experimentally measured surface energies of the DLC layer and the monolayer lubricant film for the surface pressure equation derived from the LJP.

The surface energies of the DLC layer and the prevailing PFPE lubricants with hydroxyl polar end groups have polar components in addition to dispersive components. The polar surface energy may partly have an electrostatic interaction that may not obey the LJP law. However, there is no experimental study to show how the polar surface energy generates a noncontact force in contrast to the dispersive component. Thus, we assume here that both the dispersive and polar surface energies affect the distance force subject to the LJP law for analytical simplicity as the same as the prior studies [11-13]. Below, we often call the surface pressure derived from the LJP law $\mathrm{vdW}$ pressure to distinguish it from the meniscus force.

In addition, an electrostatic force caused by an induced electric potential difference between the head and the disk surface has been often reported to have a substantial attractive force $[12,34]$. However, today's head sliders, which have an embedded contact sensor beneath the head surface, can control the electric potential difference by applying a bias voltage. Therefore, the electrostatic force is ignored in this analysis.

If we use the mechanical property values of the DLC layer (composite Young's modulus $E=133 \mathrm{GPa}$ and hardness $H=25$ GPa), referring to Katta et al. [35], and the asperity parameter values ( $\mathrm{rms}$ asperity height $\sigma_{a}=0.2 \mathrm{~nm}$ and mean asperity radius $R_{a}=15 \mathrm{~nm}$ ), referring to the measured values [4], we can obtain the plasticity index $\Psi=\left(\sigma_{a} / R_{a}\right)^{1 / 2}(E / H)=0.61$. Because the effective Young's modulus is estimated to be lower than this value because of a bonded lubricant layer, as will be discussed later, we assume that the head-disk contact is within the elastic contact regime. The elastic contact pressure is calculated from the statistical sum of Hertzian asperity contact forces based on the GW model with isotropic Gaussian asperity height distribution [17]. The validity of this model was confirmed by the author's statistical analysis of the measured surface textures for recent perpendicular recording disks [4].

2.3 Formulation of adhesion surface pressure and surface force

The vdW attractive force of an asperity acting on a flat surface with a distance $d_{a f}$ is derived by integrating the surface pressure between two flat surfaces over a spherical asperity surface with a radius $R_{a}$, which is written as

$$
F_{a}\left(d_{a f}\right)=\frac{A R_{a}}{6 d_{a f}{ }^{2}}\left\{1-\frac{1}{4}\left(\frac{z_{0}}{d_{a f}}\right)^{6}\right\}=\frac{8 \pi R_{a} \Delta \gamma}{3}\left(\frac{z_{0}}{d_{a f}}\right)^{2}\left\{1-\frac{1}{4}\left(\frac{z_{0}}{d_{a f}}\right)^{6}\right\}
$$

Here, $A$ is the Hamaker constant, $\Delta \gamma$ is the work of adhesion, and $z_{0}$ is the molecular equilibrium distance. However, instead of using the Hamaker constant given by the Lifshitz theory, we 
employ the experimentally measured surface energy of the DLC layer and the PFPE lubricant.

If we define $P_{c D L C}$ and $P_{n c D L C}$ as the vdW pressures from the DLCSs of the asperities with and without contact between the head surface and the BLS, respectively, they are written as

$$
\begin{aligned}
& P_{c D L C}\left(d_{a}\right)=\int_{d_{a}-z_{0}}^{\infty} \frac{16 \pi z_{0}^{2} R_{a} \gamma_{D L C} \rho}{3\left(z_{0}+T_{b l}\right)^{2}}\left\{1-\frac{1}{4}\left(\frac{z_{0}}{z_{0}+T_{b l}}\right)^{6}\right\} \phi_{a}\left(z_{a}\right) d z_{a} \\
& P_{n c D L C}\left(d_{a}\right)=\int_{-\infty}^{d_{a}-z_{0}} \frac{16 \pi z_{0}^{2} R_{a} \gamma_{D L C} \rho}{3\left(d_{a}-z_{a}+T_{b l}\right)^{2}}\left\{1-\frac{1}{4}\left(\frac{z_{0}}{d_{a}-z_{a}+T_{b l}}\right)^{6}\right\} \phi_{a}\left(z_{a}\right) d z_{a}
\end{aligned}
$$

Here, we assume that asperity contact occurs when $z_{a}$ approaches $d_{a}-z_{0}$. $R_{a}$ is the mean asperity radius, $\rho$ is the asperity density. The work of adhesion, $\Delta \gamma$, is given by twice the total measured surface energy of the DLC layer, $\gamma_{D L C}$ because the properties of the head and disk surfaces are very similar to each other.

The vdW pressure, $P_{n c L}$, from the lubricant film on the asperities without contact with the head surface is given by

$$
P_{n c L}\left(d_{a}\right)=\int_{-\infty}^{d_{a}-T_{m l}-z_{0}} \frac{16 \pi z_{0}^{2} R_{a} \gamma_{L} \rho}{3\left(d_{a}-z_{a}-T_{m l}\right)^{2}}\left\{1-\frac{1}{4}\left(\frac{z_{0}}{d_{a}-z_{a}-T_{m l}}\right)^{6}\right\} \phi_{a}\left(z_{a}\right) d z_{a}
$$

When the head comes into contact with the mobile lubricant, a toe-dipping meniscus is assumed to form and a meniscus adhesion force $4 \pi R_{a} \gamma_{L}$ is generated. It is considered that the meniscus adhesion force does not change until the head surface comes into contact with the BLS. Therefore, the adhesion pressure, $P_{c L}$, of the lubricant on the asperities in contact with the head surface $\left(z_{a} \geq d_{a}-T_{m l}-z_{0}\right)$ is given by

$$
P_{c L}\left(d_{a}\right)=\int_{d_{a}-T_{m l}-z_{0}}^{\infty} 4 \pi R_{a} \gamma_{L} \rho \phi_{a}\left(z_{a}\right) d z_{a}
$$

Note that Eq. (5) includes vdW pressure from the bonded lubricant when $T_{m l}=0$. The elastic contact pressure, $P_{e l}$, due to Hertzian contact between the BLS and the head surface is given by [17]

$$
P_{e l}\left(d_{a}\right)=\frac{4}{3} E^{*} R_{a}^{1 / 2} \rho \int_{d_{a}-z_{0}}^{\infty}\left(z_{a}-d_{a}+z_{0}\right)^{3 / 2} \phi_{a}\left(z_{a}\right) d z_{a}
$$

Thus, the total adhesion pressure, $P_{a d}\left(d_{a}\right)$, and total surface pressure, $P_{s}\left(d_{a}\right)$, including $P_{e l}\left(d_{a}\right)$, become respectively

$$
\begin{aligned}
& P_{a d}\left(d_{a}\right)=P_{n c D L C}\left(d_{a}\right)+P_{c D L C}\left(d_{a}\right)+P_{n c L}\left(d_{a}\right)+P_{e l}\left(d_{a}\right) \\
& P_{s}\left(d_{a}\right)=P_{e l}\left(d_{a}\right)-P_{n c D L C}\left(d_{a}\right)-P_{c D L C}\left(d_{a}\right)-P_{n c L}\left(d_{a}\right)-P_{e l}\left(d_{a}\right)
\end{aligned}
$$

The adhesion force $F_{a d}$ and surface force $F_{s}$ applied to the spherical head surface shown in Fig. 1(a) are respectively given by

$$
\begin{aligned}
& F_{a d}\left(d_{b l}\right)=2 \pi R \int_{d_{b}}^{\infty} P_{a d}\left(d_{a}\right) \mathrm{d} d_{a} \\
& F_{s}\left(d_{b l}\right)=2 \pi R \int_{d_{b}}^{\infty} P_{s}\left(d_{a}\right) \mathrm{d} d_{a}
\end{aligned}
$$

\section{Calculated results}

3.1 Various attractive pressures and elastic pressure

In the numerical calculation, we used the following measured texture parameters [4]: $z_{m}=0.5 \mathrm{~nm}, \sigma_{a}=0.2 \mathrm{~nm}, R_{a}=$ $15 \mathrm{~nm}$, and $\rho=5050 \mu \mathrm{m}^{-2}$. The surface energies of the DLC layer and the lubricant, including dispersion and polar components, were $\gamma_{D L C}=55 \mathrm{~mJ} / \mathrm{m}^{2}$ and $\gamma_{L}=25 \mathrm{~mJ} / \mathrm{m}^{2}$, respectively, referring to the measured values for the Z-Tetraol lubricant film reported by Tani et al. [33]. The molecular equilibrium distance $z_{0}$ was assumed to be $0.165 \mathrm{~nm}$ [32]. According to Katta et al. [35], the Young's modulus and Poisson's ratio of the DLC layer are estimated to be $250 \mathrm{GPa}$ and 0.24 , respectively. Therefore, the composite Young's modulus for the DLC-DLC contact was 133 GPa. When the DLC layer is covered with a bonded lubricant layer, it is expected that the effective composite Young's modulus would be reduced to some extent. If an asperity with a radius of $15 \mathrm{~nm}$ is deformed by $\sigma_{a}=0.2 \mathrm{~nm}$, Toresca's maximum shear stress occurs at a depth of $0.83 \mathrm{~nm}$ [36]. Thus, a bonded lubricant layer with a $0.5-1.2 \mathrm{~nm}$ thickness will produce a stress relaxation effect and reduce the effective composite Young's modulus. Therefore, we used an estimated composite Young's modulus $E^{*}=100 \mathrm{GPa}$ in the following numerical calculation.

Figures 2(a), (b), and (c) show $P_{n c D L C} P_{c D L C} P_{n c L}, P_{c L}$ and $P_{e l}$ as functions of separation $d_{a} / \sigma_{a}$ from the BLS for various values of $T_{L}$ when $B R=0,1$, and 0.85 , respectively. When $B R=0$, shown in Fig. 2(a), $P_{n c D L C}$ and $P_{c D L C}$ take the highest values because the separation from the BLS is the same as the separation from the DLCS. $P_{n c L}$ and $P_{c L}$ show curves similar to those of $P_{n c D L C}$ and $P_{c D L C}$, respectively, at $T_{b l}=0$, with an amount reduced by $\gamma_{L} / \gamma_{D L C}$ and shifted toward the right by $T_{L} / \sigma_{a}$. Therefore, we note that the adhesion pressure due to the lubricant becomes dominant compared to that due to the DLC layer when $T_{L} \geq 0.5$ $\mathrm{nm}$. Because the contact pressure does not change with $B R, P_{e l}$ is plotted in only Fig. 2(a).

When $B R=1$, shown in Fig. 2(b), $P_{n c L}$ and $P_{c L}$ take the lowest

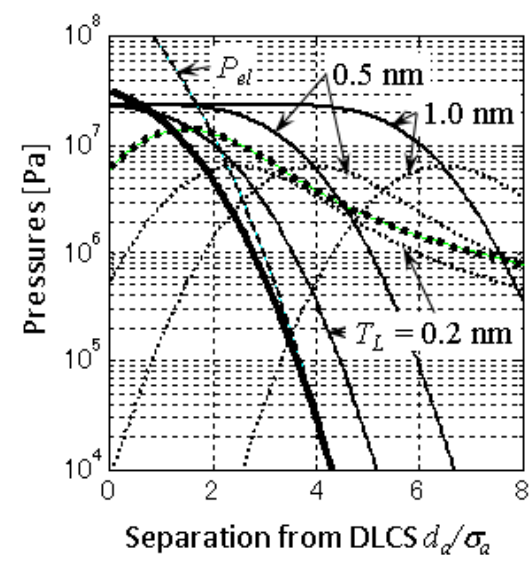

(a) $B R=0$

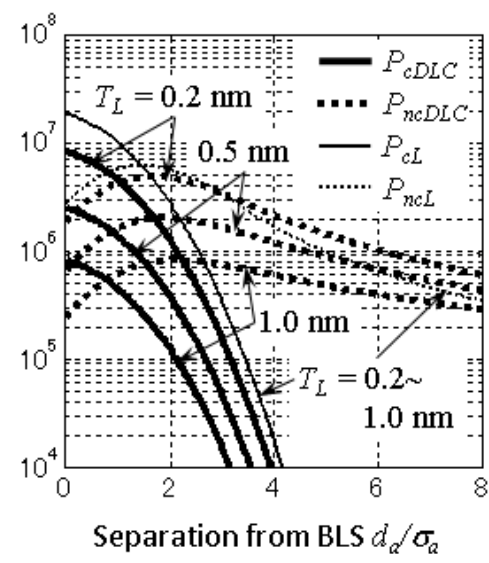

(b) $B R=1$

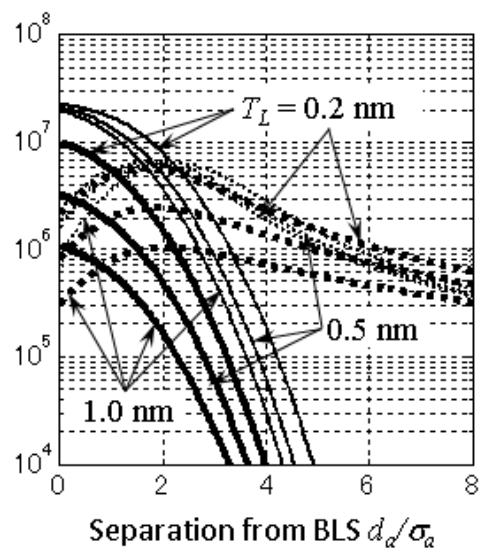

(c) $B R=0.85$

Fig. 2 Various pressures $P_{n c D L C}, P_{c D L C}, P_{n c L}, P_{c L}$ and $P_{e l}$ as a function of separation from BLS $d_{a} / \sigma_{a}$ for different lubricant layer thicknesses $T_{L}$ and bond ratios: (a) $B R=0$, (b) $B R=1.0$, (c) $B R=0.85$ 
values. We note that $P_{n c D L C}$ and $P_{C D L C}$ can be drastically reduced by increasing $T_{L}\left(=T_{b l}\right)$. When $T_{L}>0.2 \mathrm{~nm}$, the noncontact and contact $\mathrm{vdW}$ pressures due to the DLC layer decrease below those due to the bonded lubricant in the region $d_{a} / \sigma_{a}<3$. This indicates the excellent ability of the bonded lubricant to reduce high vdW pressures due to the DLC layer.

When $B R=0.85$ (the bonding ratio in current magnetic disks), with increasing $T_{L}, P_{n c D L C}$ and $P_{c D L C}$ decrease significantly, whereas $P_{n c L}$ and $P_{c L}$ increase slightly. $P_{n c D L C}$ at $T_{L}=0.2 \mathrm{~nm}$ is close to $P_{n c L}$ in the region $1<d_{a} / \sigma_{a}<4$, but it decreases rapidly as $T_{L}$ increases further. Thus, $P_{n c L}$ and $P_{c L}$ become dominant, and the sum of $P_{c L}$ and $P_{n c L}$ increases slightly as $T_{L}$ increases from 0.2 $\mathrm{nm}$.

\subsection{Adhesion pressure $P_{a d}$ and surface pressure $P_{s}$}

The total adhesive pressure $P_{a d}$ in Eq. (7) and total surface pressure $P_{s}$ in Eq. (8) versus $d_{a} / \sigma_{a}$ at $B R=0,1$, and 0.85 are plotted in Figs. 3(a), (b), and (c), respectively. When $B R=0$ with only the mobile lubricant, $P_{a d}$ at $T_{L}=0 \mathrm{~nm}$ is caused only by the $\mathrm{vdW}$ pressures of the contact and noncontact DLC layer. Note that the abscissa corresponds to the separation from the DLCS. As $T_{L}\left(=T_{m l}\right)$ increases, a noncontact vdW force and a contact meniscus force of the mobile lubricant are added to the DLC layer adhesion pressure. Therefore, $P_{a d}$ and the negative value of $P_{s}$ drastically increase and appear from a larger separation by $T_{L} / \sigma_{a}$. When $B R=1$ with only the bonded lubricant film $\left(T_{b l}\right.$ $\left.=T_{L}\right), P_{a d}$ and the maximum adhesion pressure of $P_{s}$ decrease with an increase in $T_{b l}\left(=T_{L}\right)$ due to the attenuation of $\mathrm{vdW}$ pressure from the DLC layer. When $T_{L}$ is increased to $1.0 \mathrm{~nm}$, the maximum adhesion pressure of $P_{s}$ is nearly one order of magnitude smaller than that when $B R=0$. This is mainly caused by the elimination of meniscus pressure $P_{c L}$.

When $B R=0.85$, we note that $P_{a d}$ and $P_{s}$ change only slightly, even when $T_{L}$ changes from 0.25 to $1.0 \mathrm{~nm}$. This is because with an increase in $T_{L}, P_{n c L}$ and $P_{c L}$ increase slightly, but $P_{n c D L C}$ and $P_{C D L C}$ decrease at the same time. This indicates that the present bonding ratio of $\sim 0.85$ is an excellent design point in terms of low sensitivity to lubricant thickness variation. Moreover, we note that the maximum adhesion pressure becomes minimum when $T_{L}=\sim 0.5 \mathrm{~nm}$. This means that the adhesion pressure can be decreased slightly by reducing the lubricant thickness to $\sim 0.5$ $\mathrm{nm}$. Therefore, we suggest that a thinner monolayer lubricant with a lower molecular weight should be developed to reduce head-medium spacing for higher recording density.

\subsection{Surface force $F_{s}$ applied to spherical head}

The TFC head surface has ellipsoidal geometry, with principal radii of curvatures of $R_{1}=\sim 7 \mathrm{~mm}$ and $R_{2}=\sim 32 \mathrm{~mm}$. The effective radius of curvature is estimated to be $R=\left(R_{1} R_{2}\right)^{1 / 2}=$ $15.0 \mathrm{~mm}$. Therefore, we discuss the total surface force $F_{s}$ applied to a sphere with a radius of $15 \mathrm{~mm}$.

Figures $4(\mathrm{a})-(\mathrm{d})$ show $F_{s}$ as a function of separation for various values of $T_{L}$ when $B R=0,0.5,0.85$, and 1.0, respectively. The upper figures depict $F_{s}$ as a function of separation $d_{b l} / \sigma_{a}$ from the BLS, whereas the lower figures depict $F_{s}$ as a function of separation $d_{m l} / \sigma_{a}$ from the mean asperity height of the MLS. The effects of lubricant thicknesses of 1.25, 1.0, 0.75, 0.5, and 0.25 $\mathrm{nm}$ are illustrated by the differently colored lines. Note that $d_{b l} / \sigma_{a}$ corresponds to the separation from the DLCS when $B R=0$.

From the upper figures in Fig. 4, we note that when $B R$ $=0$, the maximum adhesion forces are nearly one order of magnitude bigger than those when $B R=1$. The adhesion force increases significantly and extends to a larger $d_{b l} / \sigma_{a}$ value due to the asperity meniscus force with an increase in $T_{L}\left(=T_{b l}\right)$. Note that the value of $d_{b l} / \sigma_{a}$ that gives the maximum adhesion force of $F_{s}$ is equal to $d_{a} / \sigma_{a}$ that makes $P_{s}=0$ in Fig. 3. This means that the total adhesion force becomes maximum when $P_{s}$ is zero at the sphere apex. When $F_{s}=0 \mathrm{mN}$ (zero air-bearing force and zero external load), the equilibrium separation $d_{b l} / \sigma_{a}$ decreases to zero or even becomes negative. This suggests that the asperity contact stress becomes severe so that wear of the head and disk will be inevitable unless the lubrication effect of the mobile lubricant is generated. The adhesion force $F$ caused by a meniscus forming around a 15-mm-radius sphere with immersed lubricant with $\gamma_{L}=25 \mathrm{~mJ} / \mathrm{m}^{2}$ is $F=4 \pi R \gamma_{L}=4.71 \mathrm{mN}$. Therefore, the maximum adhesion force of $F_{s}$ at $T_{m l}=T_{L}=1.25$ $\mathrm{nm}$ is $71 \%$ of the immersed meniscus force. The maximum adhesion forces at $T_{L}=1.5$ and $2.0 \mathrm{~nm}$ are 3.95 and $5.02 \mathrm{mN}$, respectively.

Here, let us consider the limitation of this asperity meniscus adhesion theory. The distance between the lowest asperity peak height $\left(-3 \sigma_{a}\right)$ and the highest one $\left(3 \sigma_{a}\right)$ is considered to be $6 \sigma_{a}$ within $99.7 \%$ of the total asperities. When $T_{L}=6 \sigma_{a}=1.2 \mathrm{~nm}$, the lubricant surface on the lowest peak would have the same height as the DLCS at the highest asperity peak. Therefore, when the head surface comes into contact with the DLCS at the highest asperity peak, an asperity meniscus can form at almost all asperities. Therefore, asperity menisci would grow to the immersed meniscus. When $T_{L}=1.2 \mathrm{~nm}$, the maximum adhesion

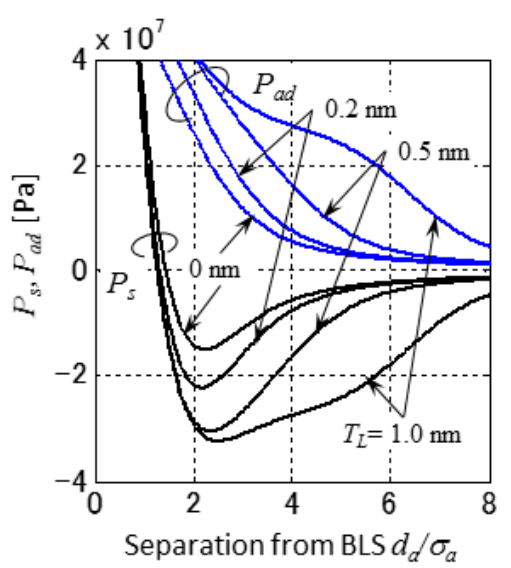

(a) $B R=0$

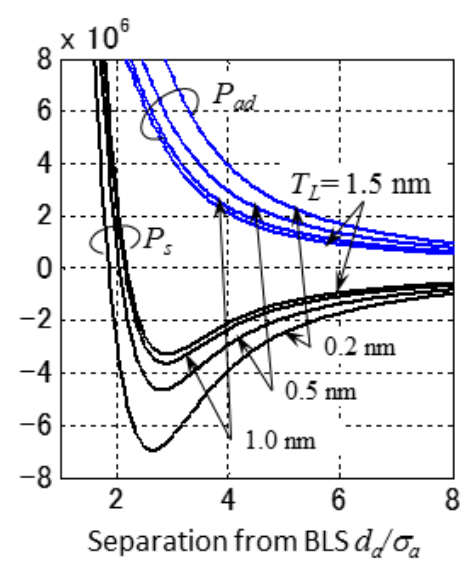

(b) $B R=1$

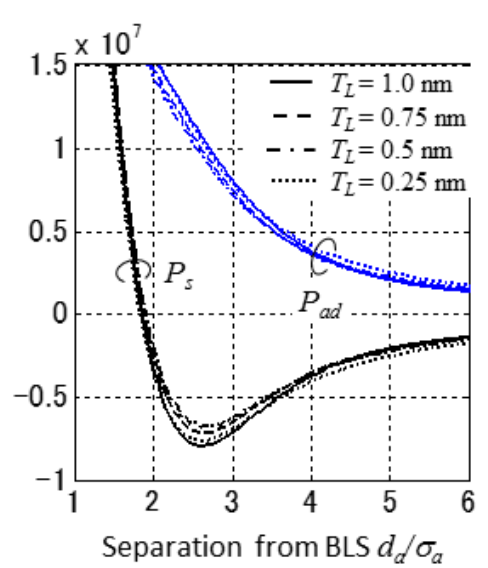

(c) $B R=0.85$

Fig. 3 Total adhesion pressure $P_{a d}$ and surface pressure $P_{s}$ as a function of sphere surface separation $d_{a} / \sigma_{a}$ for different lubricant layer thicknesses $T_{L}$ and bond ratios: (a) $B R=0$, (b) $B R=1.0$, (c) $B R=0.85$ 


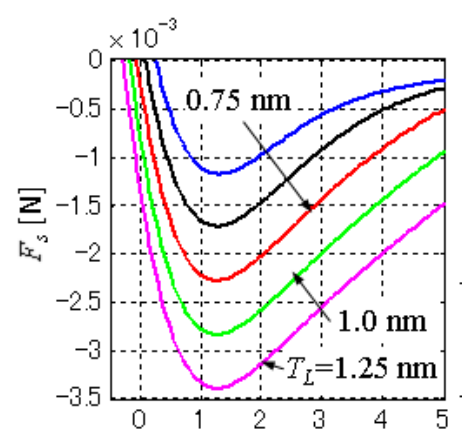

Separation from BLS, $d_{b} / \sigma_{a}$

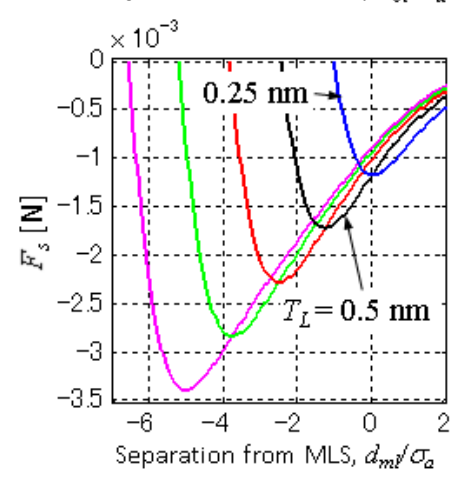

(a) $B R=0$

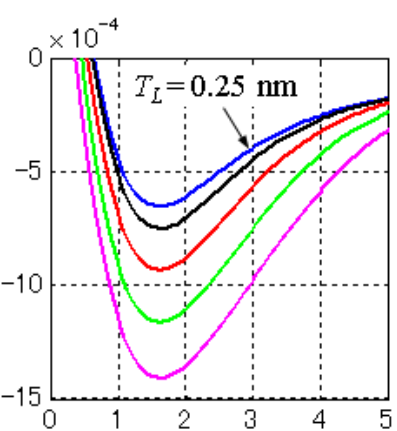

Separation from BLS, $d_{b l} / \sigma_{a}$

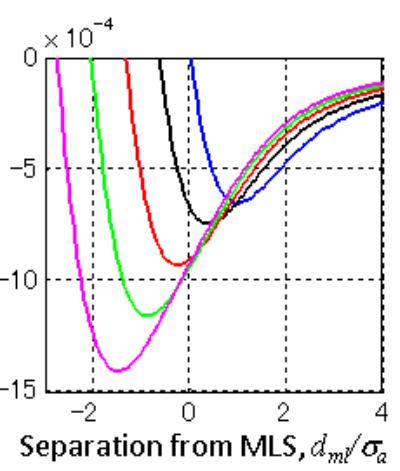

(b) $B R=0.5$

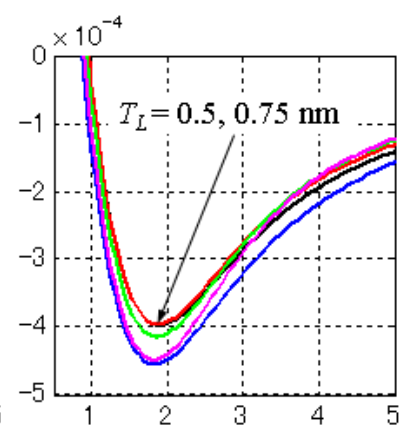

Separation from BLS $d_{b y} \sigma_{a}$

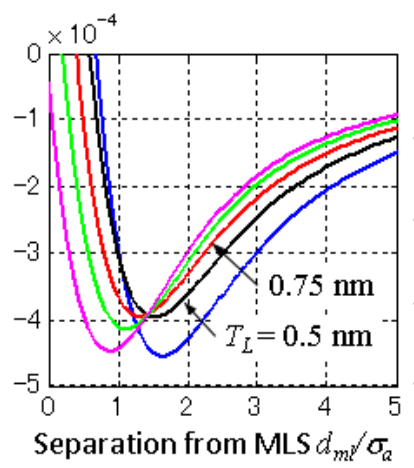

(c) $B R=0.85$
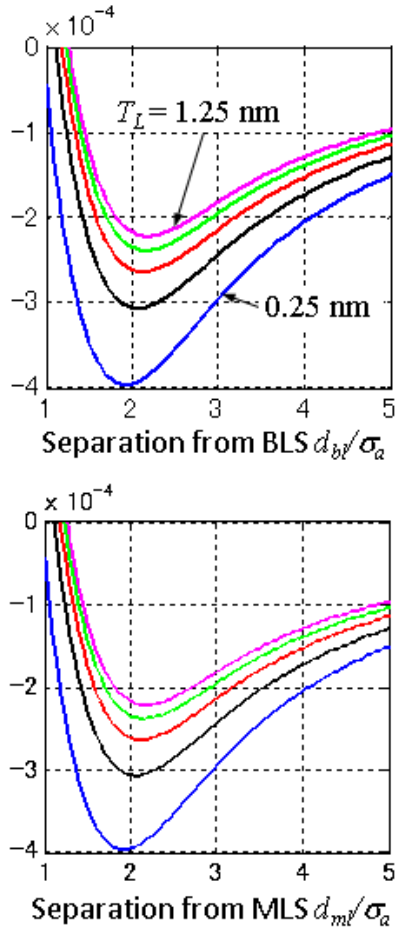

(d) $B R=1$

Fig. 4 Surface force $F_{s}$ as a function of separation from the BLS and MLS for various lubricant thicknesses and bond ratios: (a) $B R=0$, (b) $B R=0.5$, (c) $B R=0.85$, (d) $B R=1$

force becomes $3.25 \mathrm{mN}$, approximately $70 \%$ of the immersed meniscus force. This indicates that the validity of this theory is limited to a lubricant thickness of approximately $6 \sigma_{a}$. However, because of the diffusion of the lubricant film, the immersed meniscus seems to be generated if $T_{L}$ becomes larger than $2-3 \sigma_{a}$ [18].

When $B R=0.5$, the adhesion force is reduced to approximately half that when $B R=0$ because the mobile lubricant thickness decreases by half and the vdW force of the DLC layer is intercepted by the bonded layer. However, the adhesion force increases with $T_{L}$ because the mobile lubricant has a dominant effect. When $B R=0.85$, however, the maximum adhesion force becomes minimum when $T_{L}=0.5-0.75 \mathrm{~nm}$. This is because the increase in adhesion force due to asperity menisci with an increase in $T_{L}$ is compensated for by the decrease in adhesion force due to the DLC layer. When $B R=1$, the adhesion force can be further reduced because the adhesion force due to asperity menisci is completely eliminated. This result is consistent with the experimental results reported by Tani et al. [37]. As $T_{L}$ increases, $F_{s}$ approaches the lowest adhesion curve caused by the bonded layer alone. From these calculated results, we can say that the present bonded ratio of 0.85 is an excellent design point from the aspect of not only securing the lubrication effect at intermittent asperity contact but also robustness against the variation of lubricant thickness.

As seen from the lower figures in Fig. 4, the adhesion force $F_{s}\left(d_{m l} / s_{a}\right)$ as a function of $d_{m l} / \sigma_{a}$ is shifted from $F_{s}\left(d_{b l} / \sigma_{a}\right)$ by $d_{m l}=d_{b l}$ - $T_{m l}$, where $T_{m l}=T_{L} \times(1-B R)$. We note that in the region of $d_{m l} l$ $\sigma_{a}>0$, the adhesion force $F_{s}$ is less than $1.2 \mathrm{mN}$ at $B R=0$ and 0.9 $\mathrm{mN}$ at $B R \leq 0.5$. This indicates that the attractive force acting on the TFC head surface is less than $1.2 \mathrm{mN}$, even when the head surface approaches the mean asperity height of the MLS at any bond ratio and lubricant thickness as far as the formation of the asperity meniscus obeys the condition assumed in this paper without any accumulation of lubricant.

It is also interesting to note that in the separation range larger than the minimum point of $F_{s^{\prime}}$ a thinner lubricant has a lower $F_{s}$ value (larger adhesion force) at any bond ratio. This feature is more prominent as $B R$ increases, although the absolute value of $F_{s}$ decreases. When $B R=1, F_{s}$ is lower as $T_{L}$ is thinner in the entire region of separation. This indicates the possibility that the surface adhesion force $F_{s}$ with a thinner lubricant film can affect slider instability at higher FH. The static instability of the flying head slider can happen when the increasing rate of adhesion force with respect to the decrement of separation $\left(-\mathrm{d} F_{s} / \mathrm{d} d_{m l}\right)$ overcomes the air-bearing stiffness. From the lower figures when $B R=0.5$ and 0.85 , the adhesive stiffness at $T_{L}=1.0 \mathrm{~nm}$ is calculated to be $-2.21 \times 10^{5} \mathrm{~N} / \mathrm{m}$ at $d_{m l} / \sigma_{a}=4(1.3 \mathrm{~nm} F H)$ and $-1.40 \times 10^{5} \mathrm{~N} / \mathrm{m}$ at $d_{m l} / \sigma_{a}=5(1.5 \mathrm{~nm}$ $F H)$, respectively. Referring to our previous papers [38, 39], the air-bearing stiffness of the trailing pad for a pico-slider (1.25 $\mathrm{mm}$ length and $1 \mathrm{~mm}$ width) is estimated to be $10^{6}$ to $2 \times 10^{6}$ $\mathrm{N} / \mathrm{m}$. Because the adhesion force and stiffness for a pico-slider would be more than several times larger than those calculated for a TFC femto-slider, it is expected that the pico-slider would become statically unstable and touchdown to the disk surface would occur from a 1-2 nm flying height. These results are consistent with those of experimental and numerical studies of the static instability of a pico-slider [28-31]. However, once a slider comes into contact with a disk, a strong adhesion force due to meniscus force is applied to the head, as seen from Figs. $4(a)$ and (b), particularly at lubricant thicknesses larger than 0.75 $\mathrm{nm}$. Therefore, we consider that the dynamic instability of the first pitch mode of a pico-slider after touchdown is governed mainly by a strong asperity meniscus force, as discussed in our papers [38-40]. 
As stated in the introduction, this theory of surface force can well evaluate the touchdown behaviors of current femto-sliders [5-7]. It was found from this theory that the static unstable region of separation from the BLS widens when the bond ratio is decreased or the mobile lubricant thickness is increased when $B R=\sim 0.85$ [5]. This result can be understood from Fig. $4(\mathrm{c})$, where the derivative of $F_{s}$ near $d_{b l} / \sigma_{a}=2.5-3.0$ or $d_{m l} / \sigma_{a}=\sim 2$ increases with an increase in $T_{L}$. A similar tendency can be seen when $B R=0.5$, shown in Fig. 4(b). Therefore, it can be explained that a thicker mobile lubricant can induce static instability of the TFC head slider and widen the unstable region of separation (FH). As seen from Fig. 4(d), static instability can be eliminated if the bond ratio is increased to 1 . The static instability can also be eliminated by increasing the air-bearing stiffness [5-7].

From Fig. 4, we note that the characteristics of $F_{s}$ change substantially between $B R=0.85$ and $B R=1$. Because the detailed characteristics of $F_{s}$ near $B R=0.85$ are important in terms of optimal design of the lubricant layer, the effect of $T_{L}$ on $F_{s}$ when $B R=0.75,0.85$, and 0.95 is comparatively shown in Figs. 5(a), (b), and (c), respectively. When $B R=0.75,0.85$, and 0.95 , we note that the values of $T_{L}$ that minimize the maximum adhesion force are $0.4 \mathrm{~nm}, 0.6 \mathrm{~nm}$, and $1.2 \mathrm{~nm}$, respectively, and the minimum adhesion forces are $-0.47,-0.38$, and $-0.28 \mathrm{mN}$, respectively. Therefore, in terms of reducing the attractive surface force, $B R=0.95$ is preferable to $B R=0.85$, but the optimal lubricant thickness increases. If we select $T_{L}=0.6 \mathrm{~nm}$ and $B R=0.95$, the minimum value of $F_{s}$ is $-0.32 \mathrm{mN}$. This is somewhat smaller than the minimum adhesion force of $-0.38 \mathrm{mN}$ at $B R=0.85$. However, the variation due to a change of $T_{L}$ increases, and so this design condition will not be better from a practical aspect.

From the aspect of both reducing head-medium spacing for higher magnetic recording density and maintaining the lowest possible surface force for head wear reliability, the calculated results suggest that the next target is to develop a lubricant whose monolayer thickness is $\sim 0.6 \mathrm{~nm}$. The validity of this conclusion should be proved experimentally, but some experimental studies have shown consistency with this result. For example, Kim et al. [41] experimentally found that a fully baked Z-tetraol multidentate (ZTMD) lubricant layer whose effective mean thickness was reduced from $0.35 \mathrm{~nm}$ to $0.15 \mathrm{~nm}$ showed wear reliability comparable to that obtained from a nominal monolayer ZTMD lubricant with a thickness greater than $0.7 \mathrm{~nm}$. They attributed this result to the oxidation and graphitization of the DLC layer. However, there is another contribution of reduced surface force by increasing the bond ratio to 1, as seen from Figs. 4(c) and (d) and Figs. 5 (a), (b), and (c). Although the dispersion energy of unbaked $0.35 \mathrm{~nm}$ and baked $0.15 \mathrm{~nm}$ ZTMD measured by the contact angle of the testing liquids was relatively large, the effective separation between the head and disk surface for the baked $0.15 \mathrm{~nm}$ ZTMD in the wear durability test must be determined by the thickest group of locally bonded film. Kobayashi et al. [42] developed a thinner monolayer lubricant with a lower molecular weight that showed better wear durability than conventional lubricants. They attributed this improved wear durability to an increase in lubricant coverage. We expect the additional contribution of the reduced surface force due to the higher bond ratio at the same lubricant thickness, resulting in a lower friction force.

\section{Analysis of surface energy versus lubricant thickness}

The relationship between surface energy and lubricant thickness is often measured experimentally. Waltman et al. [43] and Tani et al. [33] expressed the dispersive surface energy versus lubricant thickness as the sum of a simple function of the lubricant thickness for the DLC layer, including the effective Hamaker constant and cut-off distance and the lubricant surface energy. Then they calculated these parameter values by curve fitting. Matsuoka et al. [44] proposed a more rigorous theory to evaluate the experimental dispersive surface energy function. However, this theory does not include the repulsive term of the LJP. Therefore, the theoretical surface energy function overestimates the experimental value in small lubricant thickness regions of less than $0.5 \mathrm{~nm}$. In addition, this theory does not consider the surface roughness, bonding ratio, and submonolayer thickness effects. On the other hand, Suh and Polycarpou [45] calculated the surface energy between the head and the disk with five layers based on vdW surface pressure, including a repulsive term, and evaluating the Hamaker constant from the Lifshitz theory. The validity of this theory in a subnanometer lubricant film is not clear because the Lifshitz theory is based on continuum medium model with a multimolecular layer. In addition, these theories ignore the surface roughness effect. Therefore, the validity of these theories is unclear when the lubricant thickness reaches a submonolayer level and is as thin as the roughness height.

For this reason, we propose here a theory to derive the surface energy function from the total adhesion pressure, $P_{a d}$. It

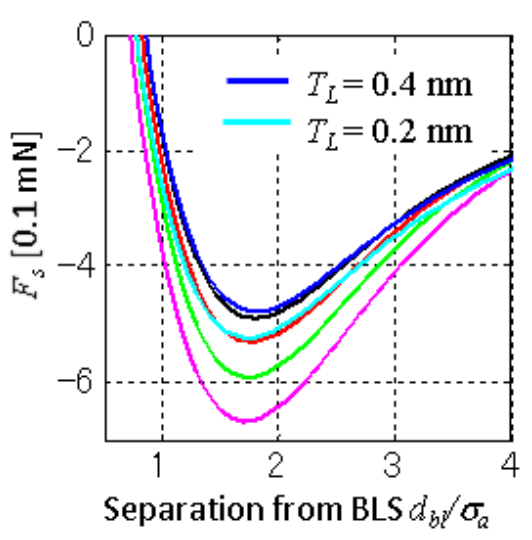

(a) $B R=0.75$

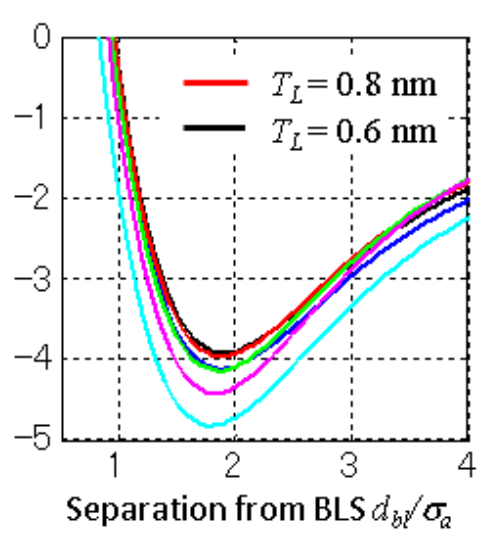

(b) $B R=0.85$

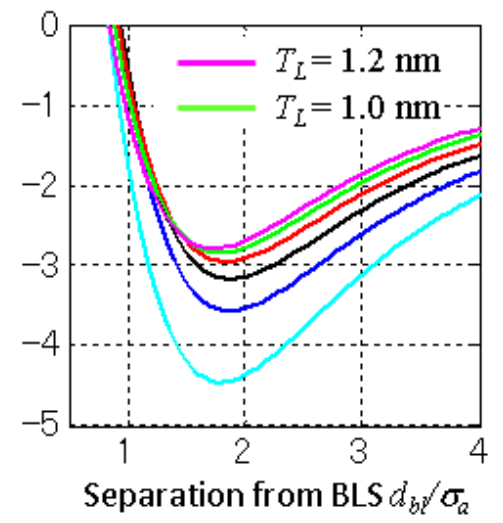

(c) $B R=0.95$

Fig. 5 Surface force $F_{s}$ as a function of separation from the BLS for various lubricant thicknesses and bond ratios: (a) $B R=0.75$, (b) $B R=0.85$, (c) $B R=0.95$ 
is considered that the surface energy of a rough DLCS coated with thin bonded and mobile lubricant layers can be obtained by integrating $P_{a d}\left(d_{a}\right)$ from infinity to contact separation $\beta \sigma_{a}$. Thus, we have

$$
\gamma\left(\beta \sigma_{a}\right)=\int_{\infty}^{\beta \sigma_{a}} P_{a d}\left(d_{a}\right) \mathrm{d} d_{a}
$$

Because the surface energy is measured by test liquids, the upper integration boundary should be the separation $d_{m l}\left(=\beta \sigma_{a}-\right.$ $\left.T_{m l}\right)$ from the mean mobile lubricant height.

Figure 6(a) shows an example of $\gamma\left(d_{m l} / \sigma_{a}\right)$ for various values of $T_{L}$ when $B R=0.85$. If we assume that the test liquid penetrating into $d_{m} / \sigma_{a}=-2.5$, we get the surface energy $\gamma(-2.5)$ from the intersection of the vertical line $d_{m l} / \sigma_{a}=-2.5$ and $\gamma\left(d_{m l} / \sigma_{a}\right)$, as shown in Fig. 6(a). Figure 6(b) depicts the surface energy versus $T_{L}$ obtained in this way for $B R=1,0.85$, and 0 . For comparison, $\gamma$ versus $T_{L}$ obtained for $d_{m l} / \sigma_{a}=-3$ is also plotted. Note that the surface energy of a lubricant such as Z03 with $B R=0$ increases gradually with decreasing $T_{L}$, whereas highly bonded lubricants such as Z-tetraol and ZTMD first increase slightly as $T_{L}$ decreases from $1.5 \mathrm{~nm}$ to $0.5 \mathrm{~nm}$ and then rapidly increase as $T_{L}$ decreases from $0.5 \mathrm{~nm}$ to $0 \mathrm{~nm}$. These results agree fairly well with the experimental data reported by Tani et al. [33] and Matsuoka et al. [44], although a detailed comparison is omitted here.

As described above, the adhesive pressure and adhesive force of this theory were calculated by using only the measured dispersive and polar surface energy values of the DLCS and the monolayer-thick lubricant film. Then, the surface energy function with respect to lubricant thickness in the submonolayer thickness region was derived theoretically. It can be said that the validity of this theory is confirmed because the theoretically derived surface energy function expresses well the experimental surface energy function throughout the lubricant thickness region of less than one monolayer.

\section{Conclusion}

Based on the adhesive asperity contact theory, the total surface pressure of multiple layers of DLC and bonded and mobile lubricant films and the total surface force applied to a thermally protruded head surface are theoretically formulated. Then, by using measured surface texture parameters and surface energy parameters of a perpendicular magnetic disk, the effects of bond ratio and lubricant thickness on the surface pressure and surface force acting on a TFC head are elucidated.
In addition, surface energy as a function of lubricant thickness and bond ratio considering the surface roughness effect is theoretically derived. The important findings of this study are summarized as follows:

(1) The bonded lubricant layer significantly suppressed the $\mathrm{vdW}$ force from the DLC layer. A bonded layer $0.2 \mathrm{~nm}$ thick decreased $P_{c D L C}$ to half the value of $P_{c L}$. A bonded layer 0.5 nm thick reduced $P_{n c D L C}$ below $P_{n c L}$ in the region $d_{a} / \sigma_{a}<5$.

(2) When $B R$ was 0.85 and the lubricant thickness was $\sim 1 \mathrm{~nm}$, the total adhesion pressure, $P_{a d}$, and surface pressure, $P_{s}$, were hardly affected by the change of $T_{L}$. This theoretically reveals that the current lubricant layer condition is the optimal robust design condition against lubricant thickness variation. The maximum value of $P_{a d}$ became minimum when $T_{L}=\sim 0.6 \mathrm{~nm}$.

(3) The maximum adhesion force applied to a 15 -mm-radius sphere when $B R=1$ and $T_{L} \geq 0.75 \mathrm{~nm}$ was nearly one order of magnitude smaller than that when $B R=0$.

(4) When $B R=0.75,0.85$, and 0.95 , the optimal $T_{L}$ values that could minimize the maximum adhesion force and provide the lowest sensitivity to lubricant thickness variations were $0.4,0.6$, and $1.2 \mathrm{~nm}$, respectively.

(5) The total adhesion force $F_{a d}$ applied to TFC head was hardly affected by the variation of $T_{L}$ when $B R=0.85$. The maximum value of $F_{a d}$ became minimum when $T_{L}=\sim 0.6 \mathrm{~nm}$ for the parameter values of the current HDI. This suggests the importance of developing lubricants thinner than $\sim 0.6$ $\mathrm{nm}$ to achieve higher recording densities.

(6) The minimum value of the surface force (maximum adhesion force) at $T_{m l}=6 \sigma_{a}$ applied to a sphere with a radius of $15 \mathrm{~mm}$ increased to $70 \%$ of the immersed meniscus force between a smooth sphere and a flat surface. Therefore, the limit of this adhesion asperity contact theory is considered to be $T_{m l} \leq \sim 6 \sigma_{a}$. However, it was estimated from experimental results that an immersed bulk meniscus can form at a much thinner mobile lubricant thickness due to rapid diffusion of the mobile lubricant around asperities. The dynamic diffusion behavior of submonolayer liquid films is one of the important subjects that remain to be studied.

(7) The relationship and consistency between the theories of static instability based on vdW pressure and dynamic instability based on asperity meniscus force proposed in studies aimed at reducing the flying height of pico-sliders to several nanometers can be understood by this theory.
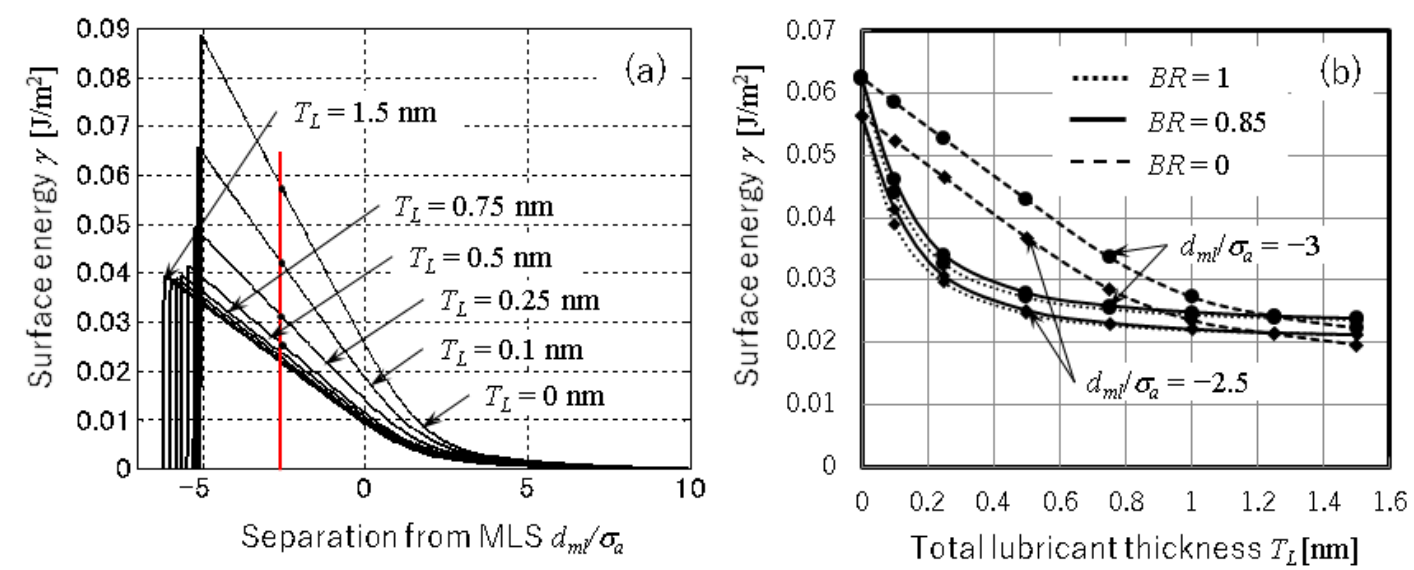

Fig. 6 (a) Surface energy at separation from mobile lubricant surface for various lubricant thicknesses and (b) surface energy at $d_{m l} / \sigma_{a}=-2.5$ and -3 versus lubricant thickness for different bond ratios 
(8) The new theory of surface energy function considering surface roughness and bond ratio agrees fairly well with the experimental data reported. This suggests the consistency and validity of this surface pressure and surface force theory.

\section{Acknowledgment}

This study was conducted with financial support from JSPS(C) 16K06039.

\section{Nomenclature}

$A=$ Hamaker constant $[\mathrm{J}]$

$B R=$ Bond ratio of lubricant $\left(=T_{m l} / T_{b l}\right)$

$E^{*}=$ Composite Young's modulus $[\mathrm{Pa}]$

$F_{a}=$ Intermolecular surface force between asperity and flat derived from Lennard-Jones potential [N]

$F_{s}=$ Surface force including the elastic contact pressure and adhesive pressures [N]

$P_{a d}=$ Total adhesive pressure from rough surface [Pa]

$P_{e l}=$ Elastic Hertzian contact pressure due to contacting asperities [Pa]

$P_{C D L C}=\mathrm{vdW}$ pressure due to DLC of contacting asperities [Pa]

$P_{c L}=$ vdW pressure due to lubricant of contacting asperities [Pa]

$P_{m e}=$ Asperity meniscus pressure due to mobile lubricant [Pa]

$P_{n c D L C}=\mathrm{vdW}$ pressure due to DLC of noncontacting asperities $[\mathrm{Pa}]$

$P_{n c L}=\mathrm{vdW}$ pressure due to lubricant of noncontacting asperities [Pa]

$P_{s}=$ Total surface pressure including all adhesive pressures and Hertzian contact pressure [Pa]

$R=$ Equivalent radius of thermally protruded head surface $[\mathrm{m}]$

$R_{a}=$ Mean radius of asperity $[\mathrm{m}]$

$T_{b l}=$ Thickness of bonded lubricant $[\mathrm{m}]$

$T_{L}=$ Total lubricant thickness $[\mathrm{m}]$

$T_{m l}=$ Thickness of mobile lubricant [m]

$d_{a}=$ Separation of local head surface from mean asperity height of bonded lubricant surface $[\mathrm{m}]$

$d_{b l}=$ Separation of head apex from mean asperity height of bonded lubricant surface $[\mathrm{m}]$

$d_{m l}=$ Separation of head apex from mean asperity height of mobile lubricant surface [m]

$h_{b l}=$ Spacing between head apex and mean surface height of BLS [m]

$h_{a}=$ Spacing between local head surface and mean asperity height of BLS [m]

$z_{a}=$ Asperity height from mean asperity height $[\mathrm{m}]$

$z_{m}=$ Mean asperity height from mean surface height $[\mathrm{m}]$

$z_{0}=$ Mean molecular equilibrium distance $[\mathrm{m}]$

$\Delta \gamma=$ Energy of adhesion at interface $\left[\mathrm{J} / \mathrm{m}^{2}\right]$

$\gamma_{D L C}=$ Sum of dispersive and polar surface energies of DLC $\left[\mathrm{J} / \mathrm{m}^{2}\right]$

$\gamma_{L}=$ Sum of dispersive and polar surface energies of lubricant $\left[\mathrm{J} / \mathrm{m}^{2}\right]$

$\rho=$ Asperity density $\left[\mu^{-2}\right]$

$\sigma_{a}=$ Root-mean-square $(\mathrm{rms})$ value of asperity height $[\mathrm{m}]$

$\phi_{a}=$ Probability density of asperity height on DLC and lubricant surfaces $\left[\mathrm{m}^{-1}\right]$

\section{References}

[1] Kurita, T., Shiramatsu, K., Miyake, A., Kato, M., Soga, H., Tanaka, S., Saegusa, S. and Suk, M., "Active Flying-Height Control Slider Using MEMS Thermal Actuator," Microsystem Technology, 12, 4, 2006, 369-375.

[2] Xu, J., Shimizu, Y., Furukawa, M., Li, J., Sano, Y., Shiramatsu, T., Aoki, Y., Matsumoto, H., Kuroki, K. and Kohira, H., “Contact/
Clearance Sensor for HDI Subnanometer Regime," IEEE Transactions on Magnetics, 50, 3, 2014, 3300205.

[3] Canchi, S. V. and Bogy, D. B., "Slider Dynamics in the LubricantContact Regime," IEEE Transactions on Magnetics, 46, 3, 2010, 764-769.

[4] Ono, K., "Surface Texture Parameters of Perpendicular Recording Magnetic Disks," IEEE Transactions on Magnetics, 53, 3, 2017, 3300806.

[5] Ono, K., "Analysis of Microwaviness-Excited Vibrations of a Flying Head Slider in Proximity and Asperity Contact," ASME Journal of Tribology, 139, 2017, 062001.

[6] Ono, K., "Numerical Analysis of Microwaviness-Excited Vibrations of Head Slider at Touchdown," Transaction of JSME, 83, 850, 2017, 17-00091 (in Japanese).

[7] Ono, K., "Numerical Analysis of Microwaviness-Excited Vibrations of a Flying Head Slider at Touchdown," Microsystem Technologies, Published online, 2018.

[8] Stanley, H. M., Etsion, I. and Bogy, D. B., "Adhesion of Contacting Rough Surfaces in the Presence of Sub-Boundary Lubrication," ASME Journal of Tribology, 112, 1, 1990, 98-104.

[9] Derjaguin, B. V., Muller, V. M. and Toporov, Y. P., "Effect of Contact Deformations on the Adhesion of Particles," J. Colloid Interface Sci., 53, 2, 1975, 314-326.

[10] Muller, V. M., Derjaguin, B. V. and Toporov, Y. P., “On Two Methods of Calculation of the Force of Sticking of an Elastic Sphere to a Rigid Plane," Colloids and Surfaces, 7, 1983, 251-259.

[11] Lee, S.-C. and Polycarpou, A. A., "Adhesion Forces for Sub-10 nm Flying-Height Magnetic Storage Head Disk Interfaces," ASME Journal of Tribology, 126, 2004, 334-341.

[12] Suh, A. Y. and Polycarpou, A. A., "Adhesive Contact Modeling for Sub-5-nm Ultralow Flying Magnetic Storage Head-Disk Interfaces Including Roughness Effects," Journal of Applied Physics, 97, 2005, 104328.

[13] Shi, X. and Polycarpou, A. A., "An Elastic-Plastic Hybrid Adhesion Model for Contacting Rough Surfaces in the Presence of Molecularly Thin Lubricant," Journal of Colloid and Interface Science, 290, 2, 2005, 514-525.

[14] Vakis, A. I., Lee, S.-C. and Polycarpou, A. A., “Dynamic HeadDisk Interface Instabilities with Friction for Light Contact (Surfing) Recording," IEEE Transactions on Magnetics, 45, 11, 2009, 4966-4971.

[15] Zheng, J. and Bogy, D. B., "Dynamic Instability of Thermal-FlyingHeight-Control Sliders at Touchdown," Microsystem Technologies, 18, 9-10, 2012, 1319-1322.

[16] Zheng, J. and Bogy, D. B., "Numerical Simulation of Touchdown Dynamics of Thermal Flying-Height Control Sliders," IEEE Transactions on Magnetics, 48, 9, 2012, 2415-2420.

[17] Greenwood, J. A. and Williamson, J. B. P., "Contact of Nominally Flat Surfaces," Proceedings of the Royal Society of London, A, 295, 1966, 300-319.

[18] Ono, K. and Nakagawa, K., "Dynamic Adhesion Characteristics of Spherical Sliders Colliding with Stationary Magnetic Disks with a Thin Lubricant Layer," Tribology Letters, 31, 2, 2008, 77-89.

[19] Marchon, B. and Karis, T. E., "Poiseuille Flow at a Nanometer Scale," Europhysics Letters, 74, 2, 2006, 294-298.

[20] Ono, K., "Replenishment Speed of Depleted Scar in Submonolayer Lubricant," Tribology Letters, 52, 2, 2013, 195-203.

[21] Mate, C. M., Payne, R. N., Dai, Q. and Ono, K., "Nanoscale Origins of Dynamic Friction in an Asymmetric Contact Geometry," Physical Review Letters, 97, 2006, 216104.

[22] Karis, T. E., Guo, X.-C. and Juang, J. Y., "Dynamics in the Bridged State of a Magnetic Recording Slider," Tribology Letters, 30, 2, 2008, 123-140. 
[23] Mate, C. M., Marchon, B., Murthy, A. N. and Kim, S.-H., “LubricantInduced Spacing Increases at Slider-Disk Interfaces in Disk Drives," Tribology Letters, 37, 3, 2010, 581-590.

[24] Ambekar, R. P. and Bogy, D. B., "Critical Clearance and Lubricant Instability at the Head-Disk Interface of a Disk Drive," Applied Physics Letters, 92, 3, 2008, 033104.

[25] Saeki, F., Fukui, S. and Matsuoka, H., "Three-Dimensional Deformation Analyses of Ultra-Thin Liquid Film (Instability Phenomena Caused by Presence of Confining Solid Surface)," Proceedings of 2007 JSME Annual Conference, 5, 2009, S1604-1-3 (in Japanese).

[26] Ono, K., "Analysis of Elastic Contact due to Meniscus Adhesion Force," Transactions of the JSME, 81, 825, 2015, 14-00673, DOI: 10.1299/transjsme.14-00673 (in Japanese).

[27] Ono, K., “Numerical Analysis of Contact Mechanics between a Spherical Slider and a Flat Disk with Low Roughness Considering Lennard-Jones Surface Forces," Journal of Adhesion Science and Technology, 24, 2010, 2341-2362.

[28] Thronton, B. H. and Bogy, D. B., "Nonlinear Aspects of Air-Bearing Modeling and Dynamic Spacing Modulation in Sub-5-nm Air Bearings for Hard Disk Drives," IEEE Transactions on Magnetics, 39, 2, 2003, 722-728.

[29] Ambekar, R., Gupta, V. and Bogy, D. B., "Experimental and Numerical Investigation of Dynamic Instability in the Head Disk Interface at Proximity," ASME Journal of Tribology, 127, 3, 2005, 530-536.

[30] Matsuoka, H., Ohkubo, S. and Fukui, S., "Corrected Expression of the van der Waals Pressure for Multilayered System with Application to Analyses of Static Characteristics of Flying Head Sliders with an Ultrasmall Spacing," Microsystem Technologies, 11, 8-10, 2005, 824-829.

[31] Tagawa, N., Mori, A. and Senoue, K., "Effects of Molecularly Thin Liquid Lubricant Films on Slider Hysteresis Behavior in Hard Disk Drives," ASME Journal of Tribology, 129, 3, 2007, 579-585.

[32] Israelachvili, J. N., "Intermolecular and Surface Forces," Second Edition, Academic Press, 1996.

[33] Tani, H., Mitsutome, T. and Tagawa, N., "Adhesion and Friction Behavior of Magnetic Disks with Ultrathin Perfluoropolyether Lubricant Films Having Different End-Groups Measured Using Pin-on-Disk Test," IEEE Transactions on Magnetics, 49, 6, 2013, 2638-2644.

[34] Knigge, B. E., Mate, C. M., Ruiz, O. and Baumgart, P. M., "Influence of Contact Potential on Slider-Disk Spacing: Simulation and Experiment," IEEE Transactions on Magnetics, 40, 4, 2004 3165-3167.

[35] Katta, R. R., Polycarpou, A. A., Lee, S. -C. and Suk, M., "Experimental and FEA Scratch of Magnetic Storage Thin-Film Disks to Correlate Magnetic Signal Degradation with Permanent Deformation," ASME Journal of Tribology, 132, 2, 2010, 021902.

[36] Johnson, K. L., “Contact Mechanics," Cambridge University Press, Cambridge, 1985, 153.

[37] Tani, H., Lu, R., Koganezawa, S. and Tagawa, N., “Ultrathin PFPE/ DLC Hybrid Overcoat for Magnetic Disks by Photoelectron-Assisted Chemical Vapor Deposition," IEEE Transactions on Magnetics, 53, 3, 2017, 3300906.

[38] Ono, K. and Yamane, M., “Experimental and Theoretical Investigation of Bouncing Vibrations of a Flying Head Slider in the Near-Contact Region," ASME Journal of Tribology, 129, 2, 2006, 246-255.

[39] Ono, K. and Yamane, M., “Erratum, Experimental and Theoretical Investigation of Bouncing Vibrations of a Flying Head Slider in the Near-Contact Region," ASME Journal of Tribology, 129, 2, 2007, 246-255, ASMR Journal of Tribology, 131, 3, 2009, 037002.

[40] Ono, K. and Yamane, M., "Analysis of Contact Characteristics of Small Rough Surfaces Taking Bulk Deformation and Meniscus Force into Consideration," ASME Journal of Tribology, 129, 3, 2007, 453-460.

[41] Kim, S. H., Guo, X. -C., Waltman, R. J., Tu, H. -B., Shatz, T. and Pocker, D. J., "Durability Against Contact Wear of Nonlubricated Disks in the Head-Disk Interface of Disk Drives," IEEE Transactions on Magnetics, 47, 1, 2011, 239-243.

[42] Kobayashi, N., Tani, H., Shimizu, T., Koganezawa, S. and Tagawa, N., "Slider Wear on Disks Lubricated by Ultra-Thin Perfluoropolyether Lubricants with Different Molecular Weights," Tribology Letters, 53, 1, 2014, 43-49.

[43] Waltman, R. J. and Guo, X. -C., "AFM Force-Distance Curves for Perfluoropolyether Boundary Lubricant Films as a Function of Molecular Polarity," Tribology Letters, 45, 2, 2012, 275-289.

[44] Matsuoka, H., Ono, K. and Fukui, S., "Study on Surface Energy of Ultra-Thin Film (Verification of Effective Dispersion Component Theory)," Journal of Advanced Mechanical Design, System, and Manufacturing, 4, 1, 2010, 391-396.

[45] Suh, A. Y. and Polycarpou, A. A., "Analytical Determination of the Surface Energy of Sub-5 nm Head-Disk Interfaces Accounting for Multilayer Effects," Journal of Applied Physics, 99, 2006, 08N111. 